\title{
The Impact of Health Insurance on Cancer Care in Disadvantaged Communities
}

Zaid M. Abdelsattar, MD ${ }^{1,2}$; Samantha Hendren, MD MPH${ }^{1}$; Sandra L. Wong, MD MS ${ }^{1,3}$

${ }^{1}$ Center for Healthcare Outcomes and Policy, University of Michigan, Ann Arbor MI

${ }^{2}$ Department of Surgery, Mayo Clinic, Rochester MN

${ }^{3}$ Department of Surgery, Dartmouth-Hitchcock Medical Center, Lebanon NH

Correspondence/Reprint requests:

Zaid M. Abdelsattar, M.D., M.Sc.

Mayo Clinic

200 First Street SW

Rochester, MN 55905

abdelsattar.zaid@mayo.edu

Phone: 507-284-8240; Fax: 507-538-7288

Funding statement: ZMA is supported by AHRQ T32 HS000053-22. SH is supported by

$\mathrm{NIH} / \mathrm{NCl} 1 \mathrm{~K} 07 \mathrm{CA} 163665-22$ and the American Society of Colon and Rectal Surgeons

Research Foundation. SLW is supported by AHRQ 1K08 HS20937-01 and American Cancer

Society RSG-12-269-01-CPHPS.

Disclosures: The authors have no disclosures to make.

Word count: Abstract: 266; Manuscript: 2,982; Pages: 26; Tables: 3; Figures: 2

Short running head: Insurance, Social Determinants \& Cancer Care

This work was presented in part as a plenary presentation at the Society of Surgical Oncology

Annual Meeting on March 27, 2015, Houston TX

This is the author manuscript accepted for publication and has undergone full peer review but has not been through the copyediting, typesetting, pagination and proofreading process, which may lead to differences between this version and the Version record. Please cite this article as doi:10.1002/ cncr.30431.

This article is protected by copyright. All rights reserved. 


\section{SYNOPSIS}

Individuals from disadvantaged communities are among millions of uninsured Americans gaining insurance under the Affordable Care Act. We examine whether health insurance and better access to care can mitigate the effects of the social determinants of health on cancer care for the leading four causes of cancer deaths in the United States.

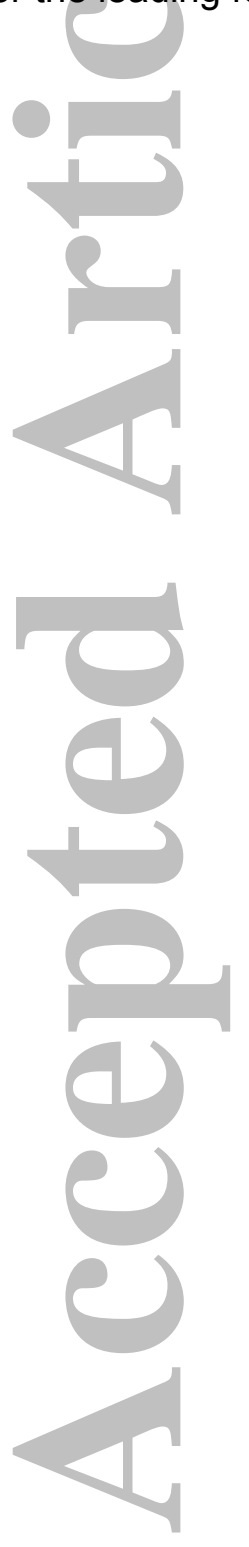




\section{STRUCTURED ABSTRACT}

PURPOSE: Individuals from disadvantaged communities are among millions of uninsured Americans gaining insurance under the Affordable Care Act. The extent to which health insurance can mitigate the effects of the social determinants of health on cancer care is unknown.

METHODS: We linked the Surveillance, Epidemiology, and End Results (SEER) registries to US-Census data to study patients diagnosed with the 4 leading causes of cancer deaths between 2007-2011. We developed a county-level social determinants score using 5 measures of wealth, education and employment. We stratified patients into quintiles, with the lowest quintile representing the most disadvantaged communities. Logistic regression and Cox proportional hazards models were used to estimate associations and cancer-specific survival. RESULTS: A total of 364,507 patients aged 18-64 years were identified $(134,105$ breast,106,914 prostate, 62,606 lung. and 60,881 colorectal). Overall, patients from the most disadvantaged communities (median household income $=\$ 42,885 ; 22 \%$ below poverty level; $17 \%$ college completion) were more likely to present with distant disease (Odds ratio [OR]=1.6; $p<0.001)$ and less likely to receive cancer-directed surgery $(O R=0.8 ; p<0.001)$ than the least disadvantaged communities (median income $=\$ 78,249 ; 9 \%$ below poverty; $42 \%$ college completion). The differences persisted across quintiles regardless of insurance status. The effect of having insurance on cancer-specific survival was more pronounced in disadvantaged communities ( $40 \%$ vs. $31 \%$ relative benefit at 3 years). However, it did not fully mitigate the effect of social determinants on mortality (Hazard Ratio 0.77 vs. $0.68 ; p<0.001$ ).

CONCLUSIONS: Cancer patients from disadvantaged communities benefit most from health insurance and there is a reduction in disparities in outcome. However, the gap produced by social determinants of health cannot be bridged by insurance alone. 


\section{INTRODUCTION}

Cancer is the second leading cause of death in the United States, and is responsible for one in every four deaths. ${ }^{1}$ Despite significant strides in overall cancer survival, several factors prevent many Americans from receiving optimal cancer care..$^{2-6}$ Individuals without health insurance lack access to health care and are more likely to be diagnosed with cancer at a later stage and have worse outcomes. ${ }^{7,8}$ The Affordable Care Act (ACA) has aimed to expand access to care, largely through the Medicaid expansion, to individuals with incomes near the national poverty levels. ${ }^{9}$ In fact, it is estimated that Medicaid will cover 93 million individuals by $2024 .^{10}$ Thus, millions of individuals from disadvantaged communities and poor social determinants of health are among those gaining health insurance.

However, it is unknown whether health insurance can mitigate the effects of the social determinants of health in patients from disadvantaged communities, and the complex interplay between these factors is not well understood. Social determinants of health are defined by the World Health Organization as the conditions in which people are born, live, learn, play, work and age. ${ }^{11}$ These community-level determinants, such as income inequality and high rates of unemployment, shape the disparities in access to health care, ${ }^{12}$ and are also associated with disproportionately lower cancer survival rates. ${ }^{13,14}$ How expanded insurance coverage will affect cancer care for Americans living in communities with varying social determinants has not been examined, and the effect of health insurance on cancer outcomes in varying social strata is unknown. . $^{4,15-17}$

In this context, we use a contemporary and nationally-representative sample of nonelderly adult patients, who are diagnosed with one of the four leading causes of cancer deaths, and explore the impact of health insurance on cancer care in different communities with varying social determinants of health. The results of this study help anticipate the effects of the ACA on cancer care and to what extent insurance mitigates the effects of the social determinants of health. 


\section{$\underline{\text { METHODS }}$}

We conducted a retrospective cohort study using the Surveillance, Epidemiology, and End Results (SEER) database. ${ }^{18}$ The SEER database is the authoritative source for cancer incidence, survival, and prevalence, currently capturing $28 \%$ of the US population, and is representative of geographic, racial, and ethnic diversity. SEER collects demographic information (e.g., age, sex, and race/ ethnicity) and clinical information (e.g., primary tumor site, tumor histology, stage, treatment, and survival) from 18 cancer registries. Institutional Review Board approval is not required for publicly available data.

Patient Population

Adult patients age 18-64 years, who were diagnosed with one of the 4 leading causes of cancer deaths (lung, female breast, prostate and colorectal), based on International Classification of Diseases for Oncology (ICD-O) site and histology codes between 1/2007 and 12/2011 were included. Patients over age 65 were excluded since most are likely to be covered by Medicare. Patients for whom this was not their first and only malignancy were excluded. SEER started collecting patient-level insurance data in January 2007 , and only released this information this past year.

Main Exposure and Stratification Variables

Insurance status was the main exposure variable. Patients were categorized as uninsured, covered by Medicaid, and insured. The "Insured" category includes those with private insurance (managed care, health maintenance organization, or preferred-provider organization), Medicare, and coverage from the military or Veterans Affairs. Patients with unknown insurance status were excluded.

We constructed a summary measure of the social determinants of health for each statecounty code using data on income (median household income; percentage of residents below the federal poverty level), education (percentage not completing high school; percentage finishing college), and occupation (percentage unemployed) from the 2008-2012 US Census 
and then linked this information to the patient's state-county code of residence in the SEER data files. The summary measure was based on previously developed methods. ${ }^{19,20}$ In brief, a zscore for each variable was estimated by subtracting the overall mean and dividing by the standard deviation (SD) for each county. Thus, a score of 2 for median household income in a county means that it is $2.0 \mathrm{SD}$ above the mean. These z-scores were then summed for each of the five variables to obtain a summary measure of the social determinants. Summary scores ranged from -21 to 10 with larger scores corresponding to a community with better social determinants. This score was used to group patients into quintiles of social determinants. The reliability (Cronbach's alpha) of this summary score was $88.3 \%$.

\section{Statistical Analyses}

In order to quantify the differences of health insurance on varying social strata, we conducted a stratified analysis. Analyses were stratified by quintiles of summary scores used to measure social determinants of health. Baseline patient characteristics across quintiles and insurance statuses were compared using logistic regression for categorical variables and oneway analysis of variance for continuous variables with the Scheffe method. ${ }^{21}$ Unadjusted associations between the different insurance groups and cancer-specific survival were displayed using Kaplan-Meier curves and compared using the log-rank test within each social determinants quintile.

A multivariable logistic regression model was used to determine the association between insurance status and receipt of cancer-directed surgery among patients with non-distant disease. The model adjusted for age, sex, race/ethnicity, marital status, cancer type, and stage. A multivariable Cox proportional hazards model, which adjusted for age, sex, race/ethnicity, cancer type, stage, and receipt of cancer-directed surgery was used to assess the effect of insurance on the endpoint of cancer-specific death for patients within each social determinants quintile. The adjusted odds ratios (aOR), adjusted hazards ratios (aHR), and their 95\%Cl's are reported. 
Due to the inherent limitation of interpreting hazard ratios between groups, ${ }^{22}$ and to facilitate the comparisons of the relative benefit of having health insurance across quintiles, a non-modeled measure was calculated by subtracting the difference of the probability of 3-year cancer-specific survival among those uninsured (i.e., control event-rate) from those insured (i.e., treated event-rate) and dividing it by the control event-rate. This relative benefit measure is akin to calculating relative risk reduction, and is easily estimated from the Kaplan-Meier curves.

All statistical tests were two-sided and p-values $<0.05$ were considered significant.

Statistical analyses were conducted using STATA special edition (version 13.1, StataCorp, College Station, TX).

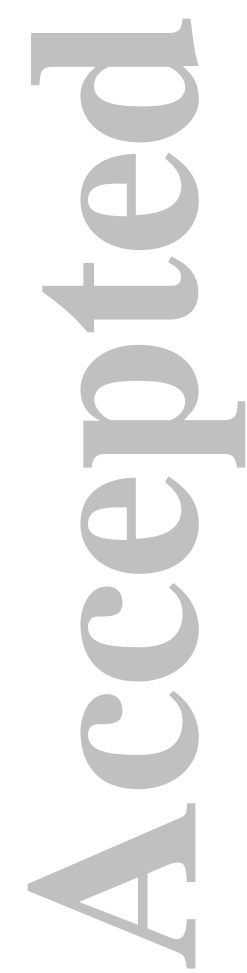




\section{$\underline{\text { RESULTS }}$}

A total of 364,507 patients between the ages of 18 and 64 years were diagnosed with one of the leading 4 causes of cancer deaths in the US between 1/2007 and 12/2011. Specifically, 134,105 (36.8\%) had breast cancer, 106,914 (29.3\%) had prostate cancer, 62,606 (17.2\%) had lung cancer, and 60,881 (16.7\%) had colorectal cancer. Of the entire cohort, 304,224 patients (83.5\%) were insured, 43,572 (12\%) had Medicaid coverage, and 16,711 $(4.6 \%)$ were uninsured.

The distributions of each of the county-level social determinants of health within each quintile are given in Table 1. For example, median household income increased in a linear fashion from $\$ 42,885$ in the most disadvantaged quintile to $\$ 78,249$ for the least disadvantaged quintile; adult residents completing college increased from $17.1 \%$ to $41.9 \%$; and employment increased from $86.7 \%$ to $92.1 \%$ respectively. Of note, poverty rates and median household incomes in the middle quintile are similar to national averages from the US census bureau for the year $2011 .^{23}$ The geographic distribution of the social determinant quintiles within SEER regions is shown in Figure 1.

The patient characteristics in the most disadvantaged and least disadvantaged communities are described in Table 2. Notably, patients in the most disadvantaged quintile were twice as likely to be uninsured $(6.0 \%$ vs. $3.0 \% ; p<0.001)$ and three-times as likely to be covered by Medicaid than those in the least disadvantaged communities $(17.5 \%$ vs. $6.7 \%$; $p<0.001)$. The average age across insurance statuses and quintiles was clinically similar to the overall cohort (54.4 \pm 7.7 years). Of note, Medicaid coverage across all quintiles was predominantly comprised of females and unmarried individuals.

Across all quintiles, lung cancer was the most common cancer among those uninsured, while breast cancer was the most common for those covered by Medicaid or those insured. Uninsured patients and those covered by Medicaid were more likely to present with distant disease than insured patients across all cancers. This effect was present within all social 
determinant quintiles. In addition, the effect of the social determinants is apparent, as insured patients from the most disadvantaged communities were still more likely to present with distant disease than insured patients from the least disadvantaged communities (17.5\% vs. $13.3 \%$; $\mathrm{p}<0.001)$

As shown in Figure 2, patients from the most disadvantaged communities have poor survival if uninsured and their cancer-specific survival markedly improves with insurance $(p<0.001)$. Medicaid insurance is associated with a modest survival benefit for patients from disadvantaged communities ( $p<0.001$ ), compared to the uninsured group, but its effect in patients from the least disadvantaged communities was negligible $(p=0.19$; Figure 2). Despite being insured, patients from the most disadvantaged quintiles still had lower cancer-specific survival when compared to insured patients from the least disadvantaged quintiles. However, the relative benefit of having insurance compared to being uninsured is more pronounced in patients from the most disadvantaged communities (40\% vs. $31 \%$ ) compared to those from the least disadvantaged communities.

Table 3 shows the adjusted effects of insurance on receipt of cancer-directed surgery and cancer-specific survival. First, health insurance is associated with higher rates of cancerdirected surgery for patients with non-distant disease and improves cancer-specific survival across all communities. Despite having health insurance, adjusting for other covariates, and considering the baseline rates, patients from the most disadvantaged communities were still less likely to receive cancer-directed surgery (aOR=1.68 vs. 1.86) and had lower cancer-specific survival $(\mathrm{aHR}=0.75$ vs. 0.68$)$ than insured patients from the least disadvantaged communities. To assess the robustness of the findings, we conducted a sensitivity analysis by each cancer type. We excluded patients with distant disease. Using a Weibull survival model, we saw insured patients from less disadvantaged communities had better cancer-specific survival than insured patients from the most disadvantaged across all cancer types. 


\section{DISCUSSION}

In this nationally representative study of patients diagnosed with one of the four leading causes of cancer deaths we sought to examine the association between having health insurance and cancer survival, for patients living in communities with varying social determinants of health. We found a consistent relationship between a community's relative advantage, measured by social determinants of health, and cancer care and outcomes, as measured by receipt of cancer-directed surgery and cancer-specific survival. Although health insurance appeared to mitigate this relationship, it did not fully abrogate the differences caused by the social determinants of health. Importantly, patients from disadvantaged communities had a larger relative benefit from health insurance, demonstrating their substantial need for improved access to care. Finally, Medicaid insurance was associated with a modest benefit on cancer survival for patients living in disadvantaged communities (compared to being uninsured), but did not have an appreciable effect in more advantaged communities.

While previous studies had shown the disparities in cancer outcomes and processes of care, ${ }^{7,24}$ to the best of our knowledge, this is the first national study to explore the interplay between health insurance and social determinants of health, and the impact on cancer care and outcomes. The findings presented herein highlight the inequities in the structure of the healthcare system in the United States and have several implications. The intent of the ACA was to provide broader coverage and better access to care for millions of Americans who are largely from socially disadvantaged communities. While this is a steppingstone in crossing the quality chasm, ${ }^{25}$ our results demonstrate that providing better access to care alone is not sufficient for equitable cancer care.

Although health insurance improved cancer care and survival across all communities, community-level social determinants significantly impacted its effectiveness. Insured patients from less disadvantaged communities still had higher odds of receiving cancer-directed surgery and better cancer-specific survival than insured patients from disadvantaged communities. This 
differential access despite health insurance is compounded by the baseline disparities between the communities.

The mechanisms underlying the persistent gap in cancer care and survival despite health insurance are unclear but probably multifactorial. Several studies have shown that patients from disadvantaged communities and minority groups may develop cancers with more aggressive biology or present with advanced disease. ${ }^{26,27}$ Other patient-related factors, including advanced age, differences in comorbid conditions, ${ }^{28}$ obesity, ${ }^{29}$ and health behaviors, such as smoking, may also influence receipt of therapy and survival. ${ }^{8}$ It is also plausible that less disadvantaged communities have better hospitals that in turn provide better care. Although not specific to cancer outcomes, Birkmeyer and colleagues ${ }^{20}$ had previously shown that disparities in surgical mortality were largely attributed to differences in the hospitals where patients received their care. This was the case for lung resection and colectomy, two procedures commonly performed for cancer. Other factors may stem directly from the public infrastructure (e.g., transportation) or the lack of social support, thereby causing a differential ability to interact with the healthcare system.

Furthermore, the increased resources in less disadvantaged communities may allow patients to absorb indirect and additional uncovered costs. These costs are particularly burdensome for cancer patients, as they have higher out-of-pocket burdens than other chronically ill patients to begin with. ${ }^{30}$ Further, patients in less disadvantaged communities might have "better" insurance plans with lower deductibles and out-of-pocket maximums by virtue of their employment benefit package, as not all insurance plans are equal. In a population-based study, Shankaran and colleagues ${ }^{31}$ found that a significant proportion of colon cancer patients undergoing adjuvant therapy experienced financial hardship despite a large proportion of patients having health insurance. In their study, $40 \%$ of patients had to sell or refinance their home, borrowed money, or experienced $>20 \%$ decline in annual income. As insurance plans increase deductibles, copayments or coinsurance fees, one can easily appreciate the prohibitive 
effect this may have on cancer care in communities with poor social determinants. The financial toxicity of cancer should be taken into consideration by cost-sharing plans as more individuals from "financially frail" communities acquire health insurance. ${ }^{32}$

In anticipating the downstream impact of the ACA, an interesting finding is that Medicaid provided a modest benefit on cancer care in disadvantaged communities, but not in less disadvantaged ones. This highlights the critical need for access to healthcare in disadvantaged communities, and provides evidence that providing Medicaid to patients in poor communities may save lives. Since we are unable to assess the timing of Medicaid coverage relative to cancer diagnosis, the effect of having regular healthcare on when cancer is diagnosed is unknown. It is possible that those in more disadvantaged communities did not have coverage until the time of diagnosis whereas those in less disadvantaged communities had the benefit of more routine healthcare antecedent to a cancer diagnosis. ${ }^{33}$ However, these findings may also suggest that Medicaid, at least in its pre-ACA form, is not sufficient. In fact, patients with Medicaid had equivalent survival to uninsured patients, in less disadvantaged communities. This may be explained by the fact that Medicaid reimburses at lower levels and is not uniformly accepted by many health care providers and institutions, ${ }^{2}$ which may be particularly true in more "affluent" hospitals. This finding may also shed light on why the published literature on the impact of Medicaid on cancer care presents mixed results. ${ }^{7}$ It may also be the case that healthcare institutions in more affluent communities provide more effective charity care to uninsured patients.

The realization that population health is in large part determined by the characteristics of the community in which they live as well as the clinical care they receive has driven many payers, hospitals and health systems to invest in stronger social support systems for their patients. However, addressing the social determinants of health and the gaps produced by them requires a coordinated effort that goes beyond the capabilities of the healthcare system alone. Rather, the pursuit of equitable cancer care should involve a multi-faceted approach including 
concerted efforts that stretch across public and private sectors and government agencies. ${ }^{34}$ Providing health insurance to the poor is an essential first step, but disadvantaged communities also require partnerships between healthcare facilities, community organizations and public health agencies. ${ }^{35}$ For example, other authors have shown that both Medicaid and uninsured patients are much more likely to present with advanced cancer, ${ }^{7}$ which further indicates that community-based cancer screening promotion might be a logical strategy in disadvantaged communities.

By intent, the social determinants summary score is based on area-level data and not patient-level socioeconomic status. The distinction between the two is important. Social determinants are shaped by the distribution of resources at the area level, and can be the target for community interventions. While it would have been ideal to assess both the community's determinants and an individual's socioeconomic status, the latter are not reported in SEER, and each has its strengths and weaknesses. For example, patient-level measures of educational attainment are erroneous in young adults because their education may not yet be complete. Similarly, area-level measures may misclassify patients on both ends of the spectrum, although this reportedly occurs at random. ${ }^{36}$ The strength of area-based measures is that they provide contextual information on the social factors that may influence cancer care for all residents of the community, which is consistent with the primary focus of this study. It is also important to acknowledge that measuring county-level social determinants blurs the reality of neighborhoodlevel experiences and may miss factors that would be significant if measured at the censuslevel.

This study has several limitations. First, patient-level insurance status in SEER is a broad classification. Nuances of covered services are unknown and the timing of insurance coverage relative to time of cancer diagnosis is unknown. Further, some uninsured patients who are diagnosed with cancer are enrolled in Medicaid either soon thereafter or at some point just prior to treatment and this is not clearly reported in the Primary Payer variable that SEER 
collects which is defined as "primary payer/insurance carrier at the time of initial diagnosis and/or treatment". Second, non-elderly patients who are insured with Medicare might have a higher proportion of disabled individuals, which may bias the results in the disadvantaged community. In addition, excluding patients $>65$ years of age may affect the external validity of the results on all cancer patients; however, the age group included in the study is the most affected by the ACA and Medicaid expansion. Further, SEER does not include data on patient functional status nor chemotherapy, which are important confounders and may proportionately differ according to social determinants.

Another important limitation in this and other studies measuring the social determinants of health, is the fact that the currently used indices for social determinants are derived using a deprivation perspective rather than a strength-based approach. Ideally, measuring adverse social determinants should be accompanied by identifying the strengths and assets of communities. A focus on community assets, opportunities and resiliencies within the build and structure of the social environment within communities would be an additional resource for health promotion. This asset-based community development (ABCD) approach is still relatively new to researchers and policy makers, but may facilitate unique interventions at the community level.

The intersection of race, poverty, and health is complex. It is evident that the most disadvantaged communities were comprised of more individuals from non-white races and ethnicities. Given the previously established racial-ethnic disparities in cancer outcomes, the associations between black race and various other indices of socioeconomic disadvantage, and the historic trust issues related to how black communities have interacted with cutting-edge cancer centers, these factors may have had an effect on the impact of health insurance in these communities. While we controlled for race/ethnicity in our models in hopes to mitigate these effects, a more in depth examination of this specific exposure goes beyond the scope of this paper. 
In conclusion, cancer patients who are from disadvantaged communities benefit most from health insurance, thereby decreasing disparities in access to care and outcomes.

However, the disparities gap produced by the social determinants of health cannot be bridged by insurance alone. As millions of Americans gain health insurance with the ACA, policy makers and payers need to keep in mind that providing healthcare insurance is necessary but not sufficient to eliminate inequities in cancer care, and substantial community-level efforts must be considered. Significant reform is needed for Medicaid to be successful in the post-ACA era.

\section{ACKNOWLEDGMENT}

Author Contributions: Drs. Abdelsattar and Wong had full access to all the data in the study and take responsibility for the integrity of the data and the accuracy of the data analysis.

Conception \& design: Abdelsattar, Wong

Data Acquisition: Abdelsattar

Analysis and interpretation: All authors

Drafting the manuscript: All authors

Critical revision: All authors

Final Approval: All authors

Conflict of Interest Disclosure: Nothing to disclose

Funding/Support: ZMA is supported by AHRQ T32 HS000053-22. SH is supported by $\mathrm{NIH} / \mathrm{NCl}$ 1 K07 CA163665-22 and the American Society of Colon and Rectal Surgeons Research Foundation. SLW is supported by AHRQ 1 K08 HS20937-01 and American Cancer Society RSG-12-269-01-CPHPS.

Role of the Sponsor: The funding source had no role in the design and conduct of the study; collection, management, analysis, and interpretation of the data; preparation, review, or approval of the manuscript; and decision to submit the manuscript for publication. 

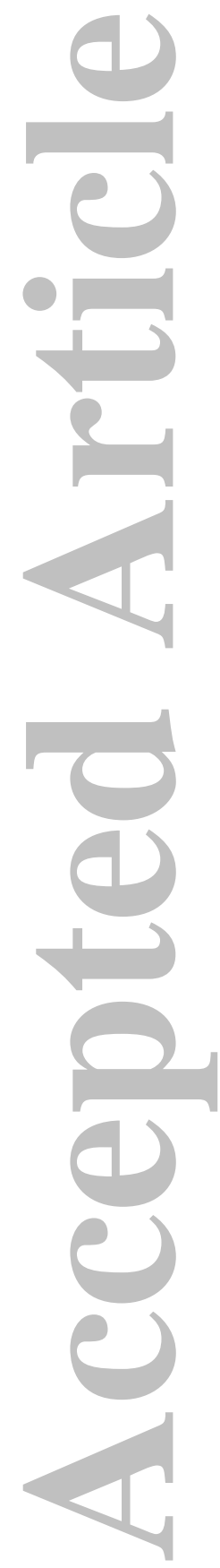

This article is protected by copyright. All rights reserved. 


\section{REFERENCES}

1. American Cancer Society. Cancer Facts \& Figures 2014. Atlanta: American Cancer Society; 2014.

2. Polite BN, Griggs JJ, Moy B, et al. American Society of Clinical Oncology Policy Statement on Medicaid Reform. J Clin Oncol. 2014:1-7. doi:10.1200/JCO.2014.56.3452.

3. Moy B, Polite BN, Halpern MT, et al. American Society of Clinical Oncology policy statement: Opportunities in the patient protection and affordable care act to reduce cancer care disparities. J Clin Oncol. 2011;29(28):3816-3824.

doi:10.1200/JCO.2011.35.8903.

4. Halpern MT, Ward EM, Pavluck AL, Schrag NM, Bian J, Chen AY. Association of insurance status and ethnicity with cancer stage at diagnosis for 12 cancer sites: a retrospective analysis. Lancet Oncol. 2008;9(3):222-31. doi:10.1016/S14702045(08)70032-9.

5. Clark CR, Soukup J, Govindarajulu U, Riden HE, Tovar D a., Johnson P a. Lack of access due to costs remains a problem for some in Massachusetts despite the state's health reforms. Health Aff. 2011;30:247-255. doi:10.1377/hlthaff.2010.0319.

6. Blackman DJ, Masi CM. Racial and ethnic disparities in breast cancer mortality: Are we doing enough to address the root causes? J Clin Oncol. 2006;24(14):2170-2178. doi:10.1200/JCO.2005.05.4734.

7. Walker G V, Grant SR, Guadagnolo BA, et al. Disparities in stage at diagnosis, treatment, and survival in nonelderly adult patients with cancer according to insurance status. $J$ Clin Oncol. 2014;32(28):3118-25. doi:10.1200/JCO.2014.55.6258.

8. Slatore CG, Au DH, Gould MK. An official American Thoracic Society systematic review: insurance status and disparities in lung cancer practices and outcomes. Am J Respir Crit Care Med. 2010;182(9):1195-205. doi:10.1164/rccm.2009-038ST.

9. Patient Protection and Affordable Care Act. Pub Law 111-148. 
0. Rudowitz R. A Look At CBO Projections For Medicaid and CHIP.; 2014.

1. World Health Organization. Social Determinants of Health. Available at: http://www.who.int/social_determinants/sdh_definition/en/.

2. Wong WF, LaVeist TA, Sharfstein JM. Achieving Health Equity by Design. JAMA. 2015;313(14):1417. doi:10.1001/jama.2015.2434.

3. Oliphant R, Nicholson G a, Horgan PG, Molloy RG, McMillan DC, Morrison DS. Deprivation and Colorectal Cancer Surgery : Longer-Term Survival Inequalities are Due to Differential Postoperative Mortality Between Socioeconomic Groups. Ann Surg Oncol. 2013;20:2132-2139. doi:10.1245/s10434-013-2959-9.

4. Byers TE, Wolf HJ, Bauer KR, et al. The impact of socioeconomic status on survival after cancer in the United States: Findings from the National Program of Cancer Registries patterns of care study. Cancer. 2008;113(June):582-591. doi:10.1002/cncr.23567.

5. Ward E, Jemal A, Cokkinides V, et al. Cancer disparities by race/ethnicity and socioeconomic status. CA Cancer J Clin. 2010;54(Figure 1):78-93.

doi:10.3322/canjclin.54.2.78, 10.3322/canjclin.54.2.78.

6. Robbins AS, Pavluck AL, Fedewa SA, Chen AY, Ward EM. Insurance status, comorbidity level, and survival among colorectal cancer patients age 18 to 64 years in the National Cancer Data Base from 2003 to 2005. J Clin Oncol. 2009;27(22):3627-33. doi:10.1200/JCO.2008.20.8025.

7. Freedman RA, Virgo KS, He Y, et al. The association of race/ethnicity, insurance status, and socioeconomic factors with breast cancer care. Cancer. 2011;117(1):180-9. doi:10.1002/cncr.25542.

8. Surveillance, Epidemiology, and End Results (SEER) Program (www.seer.cancer.gov) SEER*Stat Database: Incidence - SEER 18 Regs Research Data, Nov 2013 Sub (19732011) - Linked To County Attributes - Total U.S., 1969-2012 Counties, National Cancer Institute. 
9. Diez Roux a V, Merkin SS, Arnett D, et al. Neighborhood of residence and incidence of coronary heart disease. N Engl J Med. 2001;345(2):99-106.

doi:10.1056/NEJM200107123450205.

0. Birkmeyer NJO, Gu N, Baser O, Morris AM, Birkmeyer JD. Socioeconomic status and surgical mortality in the elderly. Med Care. 2008;46(9):893-9. doi:10.1097/MLR.0b013e31817925b0.

1. Scheffe H. The Analysis of Variance. New York: Wiley; 1959.

2. Uno $\mathrm{H}$, Claggett $\mathrm{B}$, Tian $\mathrm{L}$, et al. Moving beyond the hazard ratio in quantifying the between-group difference in survival analysis. J Clin Oncol. 2014;32(22):2380-2385. doi:10.1200/JCO.2014.55.2208.

3. Boniface CJ, Davis KE, Davis M, et al. Income and Poverty in the United States : 2013 Current Population Reports. 2014;(September).

4. Grant SR, Walker G V, Guadagnolo BA, Koshy M, Allen PK, Mahmood U. Variation in insurance status by patient demographics and tumor site among nonelderly adult patients with cancer. Cancer. 2015;121(12):2020-8. doi:10.1002/cncr.29120.

5. Baker A. Crossing the Quality Chasm A New Health System for the 21st Century.; 2001. doi:10.1136/bmj.323.7322.1192.

6. Booth CM, Li G, Zhang-Salomons J, Mackillop WJ. The impact of socioeconomic status on stage of cancer at diagnosis and survival: a population-based study in Ontario, Canada. Cancer. 2010;116(17):4160-7. doi:10.1002/cncr.25427.

7. lqbal J, Ginsburg O, Rochon PA, Sun P, Narod SA, Li Cl. Differences in Breast Cancer Stage, Treatment, and Survival by Race and Ethnicity. JAMA. 2015;163(2):49. doi:10.1001/jama.2014.17322.

8. Bickell N a, Wang JJ, Oluwole S, et al. Missed opportunities: racial disparities in adjuvant breast cancer treatment. J Clin Oncol. 2006;24(9):1357-1362.

doi:10.1200/JCO.2005.04.5799. 
9. Chan DSM, Vieira AR, Aune D, et al. Body mass index and survival in women with breast cancer-systematic literature review and meta-analysis of 82 follow-up studies. Ann Oncol. 2014;25(10):1901-14. doi:10.1093/annonc/mdu042.

0. Bernard DSM, Farr SL, Fang Z. National estimates of out-of-pocket health care expenditure burdens among nonelderly adults with cancer: 2001 to 2008 . J Clin Oncol. 2011;29(20):2821-2826. doi:10.1200/JCO.2010.33.0522.

1. Shankaran V, Jolly S, Blough D, Ramsey SD. Risk factors for financial hardship in patients receiving adjuvant chemotherapy for colon cancer: a population-based exploratory analysis. J Clin Oncol. 2012;30(14):1608-14.

doi:10.1200/JCO.2011.37.9511.

2. Zafar SY, Peppercorn JM, Schrag D, et al. The financial toxicity of cancer treatment: a pilot study assessing out-of-pocket expenses and the insured cancer patient's experience. Oncologist. 2013;18:381-90. doi:10.1634/theoncologist.2012-0279.

3. Halpern MT, Romaire MA, Haber SG, Tangka FK, Sabatino SA, Howard DH. Impact of state-specific Medicaid reimbursement and eligibility policies on receipt of cancer screening. Cancer. 2014;120(19):3016-24. doi:10.1002/cncr.28704.

4. Weil AR. It Takes A Community. Health Aff. 2014;33(11):1886-1886. doi:10.1377/hlthaff.2014.1092.

5. Rogerson B, Lindberg R, Givens M, Wernham A. A Simplified Framework For Incorporating Health Into Community Development Initiatives. Health Aff. 2014;33:1939_ 1947. doi:10.1377/hlthaff.2014.0632.

6. Subramanian S V, Chen JT, Rehkopf DH, Waterman PD, Krieger N. Comparing individual- and area-based socioeconomic measures for the surveillance of health disparities: A multilevel analysis of Massachusetts births, 1989-1991. Am J Epidemiol. 2006;164(9):823-34. doi:10.1093/aje/kwj313. 


\section{LEGEND}

FIGURE 1: Map of the United States showing the geographic distribution of the social determinants quintiles across communities within the 18 SEER regions. Darker colors correspond to communities with greater social disadvantage.

FIGURE 2: Kaplan-Meier curves for cancer specific survival by insurance status for patients living in the most disadvantaged communities (A), compared to patients living in the least disadvantaged communities (B). Note that insured patients in $\mathbf{A}$, have worse survival than insured patients in $\mathbf{B}$. However, the relative survival benefit from health insurance at 3 years is greater for patients from disadvantaged communities compared to those less disadvantaged (40\% vs. $31 \%)$.

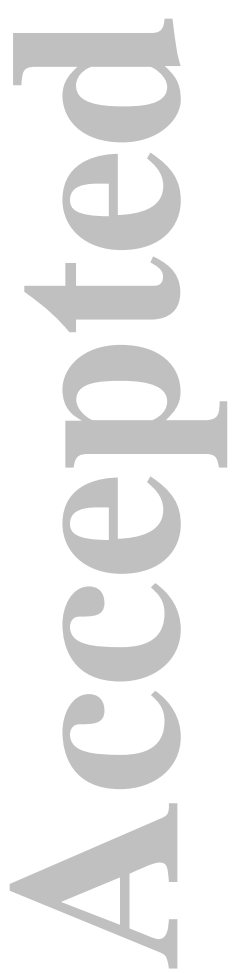




\begin{tabular}{|c|c|c|c|c|c|}
\hline \multirow[b]{2}{*}{ Area-level Social Determinants of Health } & \multicolumn{5}{|c|}{ Social Determinants Quintile } \\
\hline & $\begin{array}{c}\text { Most } \\
\text { disadvantaged }\end{array}$ & 2 & Middle & 4 & $\begin{array}{c}\text { Least } \\
\text { disadvantaged }\end{array}$ \\
\hline No. of patients & 79,019 & 69,990 & 70,456 & 72,788 & 72,254 \\
\hline Mean summary score & -6.1 & -1.8 & 0.68 & 2.7 & 5.1 \\
\hline \multicolumn{6}{|l|}{ Wealth/Income } \\
\hline Median household income (\$) & $\$ 42,885$ & $\$ 53,008$ & $\$ 55,891$ & $\$ 67,301$ & $\$ 78,249$ \\
\hline Persons below federal poverty level (\%) & $21.9 \%$ & $16.9 \%$ & $14.1 \%$ & $11.6 \%$ & $8.6 \%$ \\
\hline \multicolumn{6}{|l|}{ Education } \\
\hline Adult residents who completed high school (\%) & $77.8 \%$ & $80.2 \%$ & $86.9 \%$ & $87.9 \%$ & $90.7 \%$ \\
\hline Adult residents who completed college (\%) & $17.1 \%$ & $26.8 \%$ & $28.0 \%$ & $34.8 \%$ & $41.9 \%$ \\
\hline \multicolumn{6}{|l|}{ Employment } \\
\hline Employed residents (\%) & $86.7 \%$ & $89.1 \%$ & $90.7 \%$ & $90.9 \%$ & $92.1 \%$ \\
\hline
\end{tabular}




\begin{tabular}{|c|c|c|c|c|c|c|}
\hline \multirow[b]{2}{*}{ Characteristics } & \multicolumn{3}{|c|}{ Most Disadvantaged Communites } & \multicolumn{3}{|c|}{ Least Disadvantaged Communities } \\
\hline & Uninsured & Medicaid & Insured & Uninsured & Medicaid & Insured \\
\hline Patients, n (\%) & $4,709(6.0 \%)$ & $13,815(17.5 \%)$ & $60,495(76.6 \%)$ & $2,174(3.0 \%)$ & $4,808(6.7 \%)$ & $65,272(90.3 \%)$ \\
\hline \multicolumn{7}{|l|}{ Age } \\
\hline Mean, years \pm SD & $53.9 \pm 7.7$ & $53.1 \pm 8.2$ & $55.1 \pm 7.3$ & $54 \pm 8.2$ & $53.2 \pm 8.2$ & $54.2 \pm 7.7$ \\
\hline \multicolumn{7}{|l|}{ Gender } \\
\hline Female & $2,189(46.5)$ & 8,552 (61.9) & $28,617(47.3)$ & $1,086(50)$ & $2,885(60)$ & $34,062(52.2)$ \\
\hline \multicolumn{7}{|l|}{ Marital Status } \\
\hline Married & $1,935(41.1)$ & $4,757(34.4)$ & $39,699(65.6)$ & 825 (37.9) & $1,470(30.6)$ & $45,419(69.6)$ \\
\hline \multicolumn{7}{|l|}{ Race and ethnicity } \\
\hline Non-hispanic white & $2,754(58.5)$ & $7,103(51.4)$ & $39,948(66)$ & $1,348(62)$ & $2,466(51.3)$ & $48,116(73.7)$ \\
\hline Non-hispanic black & $1,338(28.4)$ & $3,611(26.1)$ & $12,393(20.5)$ & $273(12.6)$ & 745 (15.5) & $4,530(6.9)$ \\
\hline Hispanic & $508(10.8)$ & $2,494(18.1)$ & $6,174(10.2)$ & $295(13.6)$ & $654(13.6)$ & $3,539(5.4)$ \\
\hline Other & $109(2.3)$ & $607(4.4)$ & $1,980(3.3)$ & 258 (11.9) & $943(19.6)$ & 9,087 (13.9) \\
\hline \multicolumn{7}{|l|}{ Tumor Type } \\
\hline Lung & $1,762(37.4)$ & 4,645 (33.6) & $11,126(18.4)$ & $631(29)$ & $1,443(30)$ & $8,245(12.6)$ \\
\hline Colorectal & $1,329(28.2)$ & $2,450(17.7)$ & $10,328(17.1)$ & $513(23.6)$ & 939 (19.5) & $9,652(14.8)$ \\
\hline Breast & $884(18.8)$ & $5,212(37.7)$ & $19,678(32.5)$ & $621(28.6)$ & $1,878(39.1)$ & 25,895 (39.7) \\
\hline Prostate & $734(15.6)$ & $1,508(10.9)$ & $19,363(32)$ & $409(18.8)$ & $548(11.4)$ & 21,480 (32.9) \\
\hline \multicolumn{7}{|l|}{ Stage at presentation } \\
\hline Distant disease (Overall) & $1,959(41.6)$ & $4,625(33.5)$ & 10,594 (17.5) & $756(34.8)$ & $1,571(32.7)$ & $8,654(13.3)$ \\
\hline Distant disease (Lung) & $1,268(72)$ & 2949 (63.5) & 6552 (58.9) & $440(69.7)$ & $936(64.9)$ & $4946(60)$ \\
\hline Distant disease (Colorectal) & $442(33.3)$ & $766(31.3)$ & $2296(22.2)$ & $181(35.3)$ & $319(34)$ & $1998(20.7)$ \\
\hline Distant disease (Breast) & $148(16.7)$ & $736(14.1)$ & $1280(6.5)$ & $92(14.8)$ & $243(12.9)$ & $1308(5.1)$ \\
\hline Distant disease (Prostate) & $101(13.8)$ & $174(11.5)$ & $466(2.4)$ & $43(10.5)$ & 73 (13.3) & 402 (1.9) \\
\hline \multicolumn{7}{|l|}{ Cancer-directed surgery } \\
\hline Received* & $1,894(68.9)$ & $6,831(74.3)$ & 38,387 (76.9) & $993(70)$ & $2,526(78)$ & 45,937 (81.1) \\
\hline
\end{tabular}

*Excludes patients with distant disease 


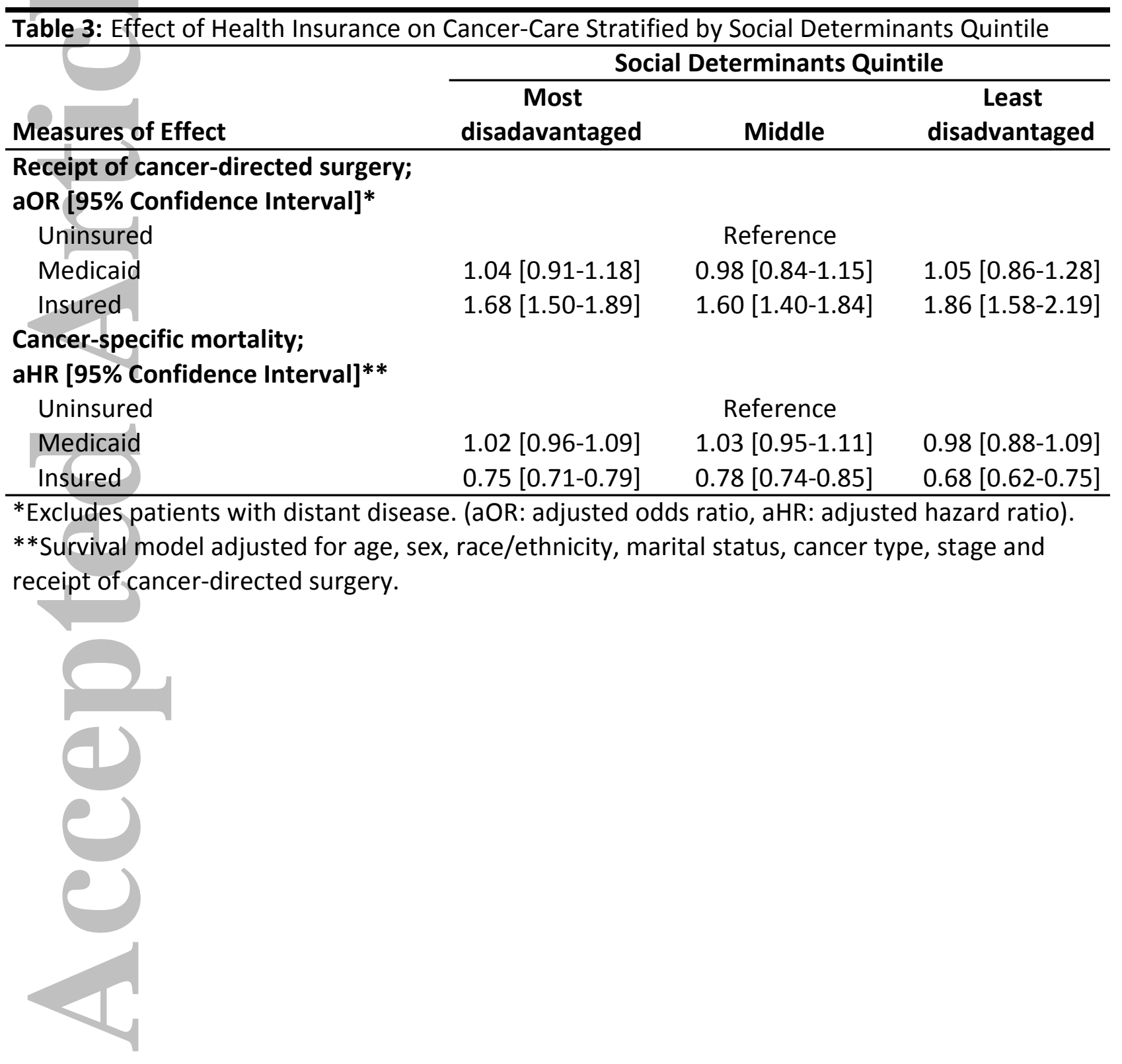




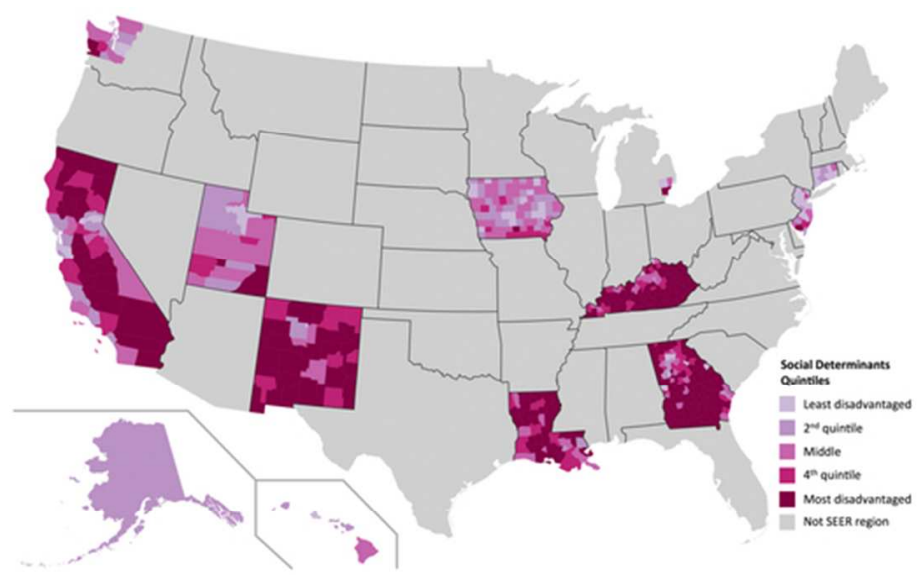

FIGURE 1: Map of the United States showing the geographic distribution of the social determinants quintiles across communities within the 18 SEER regions. Darker colors correspond to communities with greater social disadvantage.

Figure 1

(Figure 1)

$31 \times 24 \mathrm{~mm}(600 \times 600 \mathrm{DPI})$

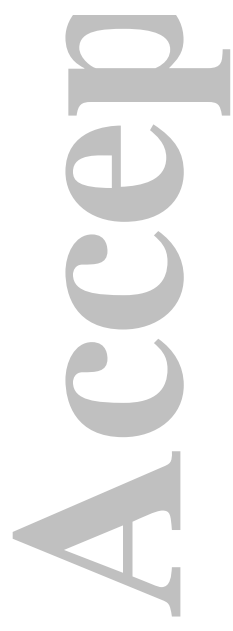

This article is protected by copyright. All rights reserved. 


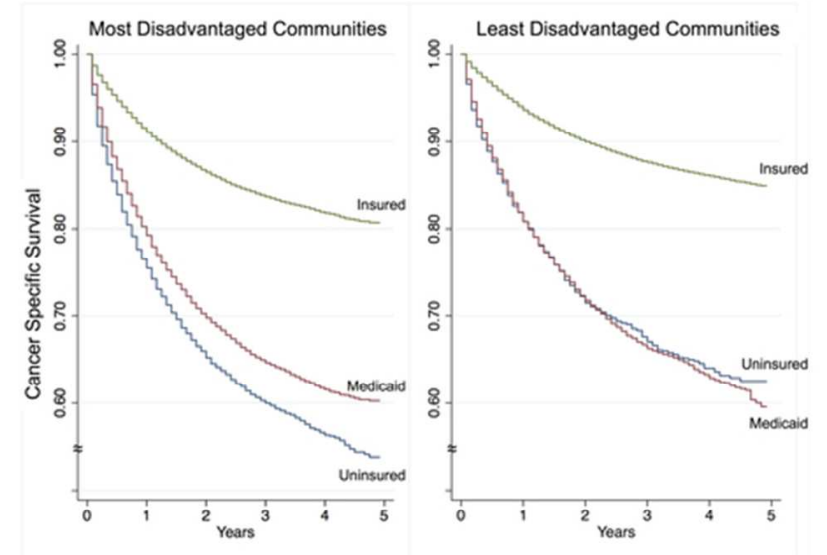

FIGURE 2: Kaplan-Meier curves for cancer specific survival by insurance status for patients living in the most disadvantaged

communities (A), compared to patients living in the least disadvantaged communities (B). Note that insured patients in $\mathbf{A}$, have worse

survival than insured patients in B. However, the relative survival benefit from health insurance at 3 years is greater for patients from

disadvantaged communities compared to those less disadvantaged ( $40 \%$ vs. $31 \%$ ).

Figure 2

(Figure 2)

$31 \times 24 \mathrm{~mm}(600 \times 600 \mathrm{DPI})$

This article is protected by copyright. All rights reserved. 